\title{
Design of Sound positioning system In the complex environment of noise based on STM32
}

\author{
Zou Long ${ }^{1,2,3}$, Taoyang Luo ${ }^{1,2,3}$, ${ }^{\text {Zhenrong Zhang }}{ }^{1,2,3}$ \\ ${ }^{1}$ School of Computer, Electronics and Information, Guangxi University, Nanning 530004, China \\ ${ }^{2}$ Guangxi Key Laboratory of Multimedia Communications and Network Technology (Cultivating \\ Base), Guangxi University, Nanning 530004, China \\ ${ }^{3}$ Guangxi Colleges and Universities Key Laboratory of Multimedia Communications and \\ Information Processing, Guangxi University, Nanning 530004, China \\ zzzr76@gxu.edu.cn(Corresponding author)
}

\begin{abstract}
Keywords: Positioning systems, spectral analysis, adaptive filters, sound
Abstract. We designed a sound localization system based STM32. Information obtained from a plurality of sound receivers for sound source localization. System consists of the sound source module, sound receiver, spectrum detector, narrow-band filter [1] and microcomputer. By looking at the spectral detector, we can choose the cleanest spectrum point in noise environment, and then choose this frequency as the frequency of the sound source to achieve localization in complex noisy environment, locating the target. The innovation lies in its ability to work in a complex environment with strong anti-jamming performance.
\end{abstract}

\section{Introduction}

From GPS to the mobile phone positioning, it is playing a more and more important role. Sound positioning determines the sound source (test target) position in space, which has been widely used in geological exploration, search rescue people, tracking and so on. Now that sound positioning is widely used in video telephony, video conferencing systems, etc.

The principle of sound localization system is that: audio signal is generated through the microcomputer, the signal is amplified through the amplifier and input to the speaker as a sound source [2]; receiving module use the microphone to receive, then amplifies the received signal, then after the band-pass filter to remove the noise of the surrounding environment, the filtered signal is just a loudspeaker sound signal. Sound source localization is through the three received signal to deal with. After a relatively perfect algorithm can get the coordinates of sound source, sound source localization can be carried out

In fact, the sound localization principle can only be used in a quiet environment, because:

(a) In a noisy environment, if the intensity of the noise is large, the sound source is likely to be covered by the noise, making the sound receiver unable to recognize the sound of the sound source.

(b)The frequency of the sound source is fixed, and if the frequency of certain noise is just equal to the frequency of the sound source, the sound receiver is unable to recognize the sound.

In order to solve above two problems, we design a sound localization system based on STM32, it can be able to work in a complex noise environment. The first is through sound receiver to receive the surrounding environment noise, and then on the STM32 FFT spectrum analysis program [3], find the frequency F, the frequency is the smallest of environmental noise points; And then let the sound source send F frequency sound waves, at the same time, adjust the frequency of the band pass filter as F; At last, analyze the three sound received by the phase of the sound signal, can calculate the sound source coordinates X, Y. New sound positioning system design block diagram in Fig. 1. 


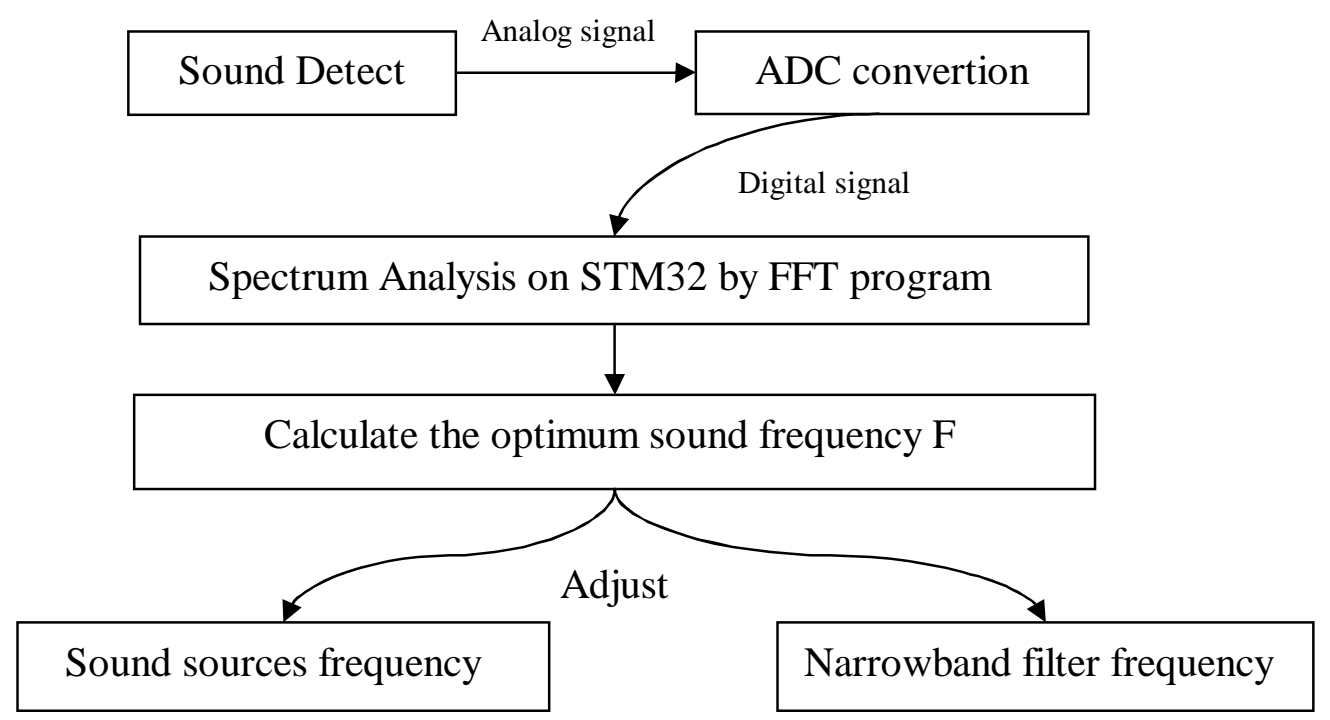

Fig. 1 Schematic of optimal frequency selection

\section{Sound localization algorithm}

Time difference (TDOA) positioning is also called hyperbolic positioning [4], is a kind of important passive location method, it is a sensor by dealing with three or more time of arrival to the collected signals, fixing the unknown source of measured data.In the two-dimensional plane, the time difference of two sensor determines the sound source signal reaches a sensor as the focus of hyperbola, using three station can form two pairs of hyperbolic intersection, recycling direction finding information to eliminate the false points, can determine the position of the sound source[5].

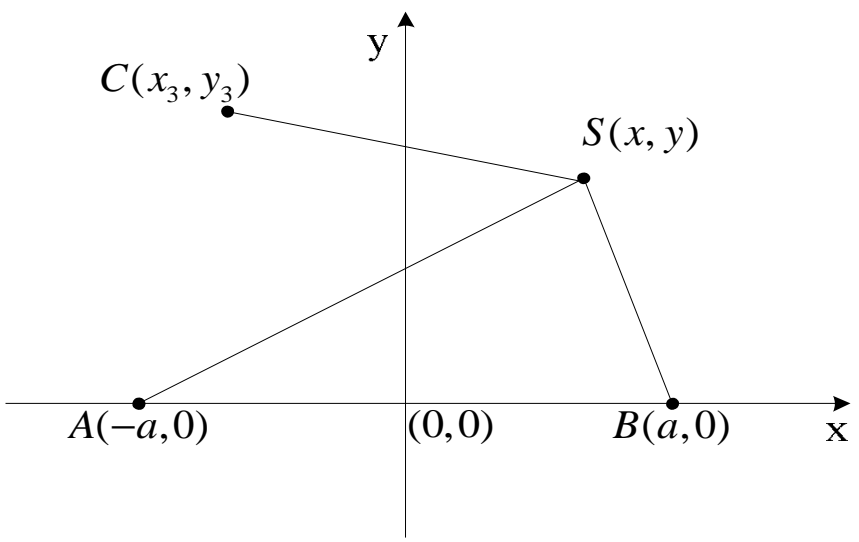

Fig. 2 The calculation diagram

As shown in Fig. 2, there are three sensors in the plane distribution. A, B and C coordinates respectively (- A, 0), $(\mathrm{A}, 0)$ and $\left(x_{3}, y_{3}\right)$. When the plan $\mathrm{S}(\mathrm{x}, \mathrm{y})$ make a sound, three sensors will successively received signal.Set signal arrived in time $\Delta t_{1}$ for the $\mathrm{A}$ and $\mathrm{B}$, reach the time $\Delta t_{2}$ difference of $\mathrm{A}$ and $\mathrm{C}$, arrived in time $\Delta t_{3}$ for the $\mathrm{B}$ and $\mathrm{C}$, the sound source location should be $\Delta t_{1} v$ in the distance to $\mathrm{A}$ and $\mathrm{B}$ two points for the curve and the distance of two points $\mathrm{A}$ and $\mathrm{C}$ is poor for curve, determine the intersection of two curve can determine the source location.It is obvious that three time general 3 hyperbola.Because there are a error in the experiment, thus three curves can't pay 
for a bit, but formed two intersecting Angle.To solve the derivation process of the 3 point coordinates are as follows.Set A (- A, 0), B (A, 0), C (x3, y3), S (x, y)。

Article 3 curve equation, respectively, as follows :

$$
\begin{aligned}
& \sqrt{(x+a)^{2}+y^{2}}+\sqrt{(x-a)^{2}+y^{2}}=\Delta t_{1} v=c_{1} \\
& \sqrt{(x+a)^{2}+y^{2}}+\sqrt{\left(x-x_{3}\right)^{2}+\left(y-y_{3}\right)^{2}}=\Delta t_{2} v=c_{2} \\
& \sqrt{(x-a)^{2}+y^{2}}+\sqrt{\left(x-x_{3}\right)^{2}+\left(y-y_{3}\right)^{2}}=\Delta t_{3} v=c_{3}
\end{aligned}
$$

By type (2-6) can be :

$$
\sqrt{(x+a)^{2}+y^{2}}=\frac{4 a x+c_{1}^{2}}{2 c_{1}}
$$

By type (2-7) can be :

$$
\sqrt{(x+a)^{2}+y^{2}}=\frac{\left(2 a+2 x_{3}\right) x+2 y_{3} y+a^{2}+c_{2}^{2}-y_{3}^{2}-x_{3}^{2}}{2 c_{2}}
$$

By type (2-9) type (2-10) can get :

$$
\frac{\left(4 a c_{2} / c_{1}-2 a-2 x_{3}\right) x+c_{1} c_{2}-a^{2}-c_{2}^{2}+y_{3}^{2}+x_{3}^{2}}{2 y_{3}}=y
$$

Make :

$$
\left\{\begin{array}{l}
m=\frac{4 a c_{2} / c_{1}-2 a-2 x_{3}}{2 y_{3}} \\
n=\frac{c_{1} c_{2}-a^{2}-c_{2}^{2}+y_{3}^{2}+x_{3}^{2}}{2 y_{3}}
\end{array}\right.
$$

Type (2-11), simplified as type plug (2-9) to go :

$$
\left(m^{2}+1-4 a^{2} / c_{1}^{2}\right) x^{2}+2 m n x+n^{2}+a^{2}-c_{1}^{2} / 4=0
$$

Make :

$$
\left\{\begin{array}{l}
d_{1}=m^{2}+1-4 a^{2} / c_{1}^{2} \\
d_{2}=n^{2}+a^{2}-c_{1}^{2} / 4
\end{array}\right.
$$

Type (2-13), simplified as :

$$
d_{1} x^{2}+2 m n x+d_{2}=0
$$

The conditions of the equation having solution :

$$
4 m^{2} n^{2}-4 d_{1} d_{2} \geq 0
$$

The final results :

$$
\left\{\begin{array}{l}
x=\frac{-2 m n+\sqrt{4 m^{2} n^{2}-4 d_{1} d_{2}}}{2 d_{1}} \\
y=\frac{-m^{2} n}{d_{1}}+\frac{m \sqrt{4 m^{2} n^{2}-4 d_{1} d_{2}}}{2 d_{1}}+n
\end{array}\right.
$$




\section{Architecture of data acquisition device}

STM32 is a family of 32-bit microcontroller integrated circuits by STMicroelectronics. Here we use STM32F407VGT6 as control chip, it is the first group of STM32 microcontrollers -based on the ARM architecture M4F core. STM32F407VGT6's core at a maximum clock rate of $168 \mathrm{MHz}$, and the capacity of the RAM is $192 \mathrm{~KB}$. Moreover, it has three 12-bit analog-to-digital converters(ADC) .

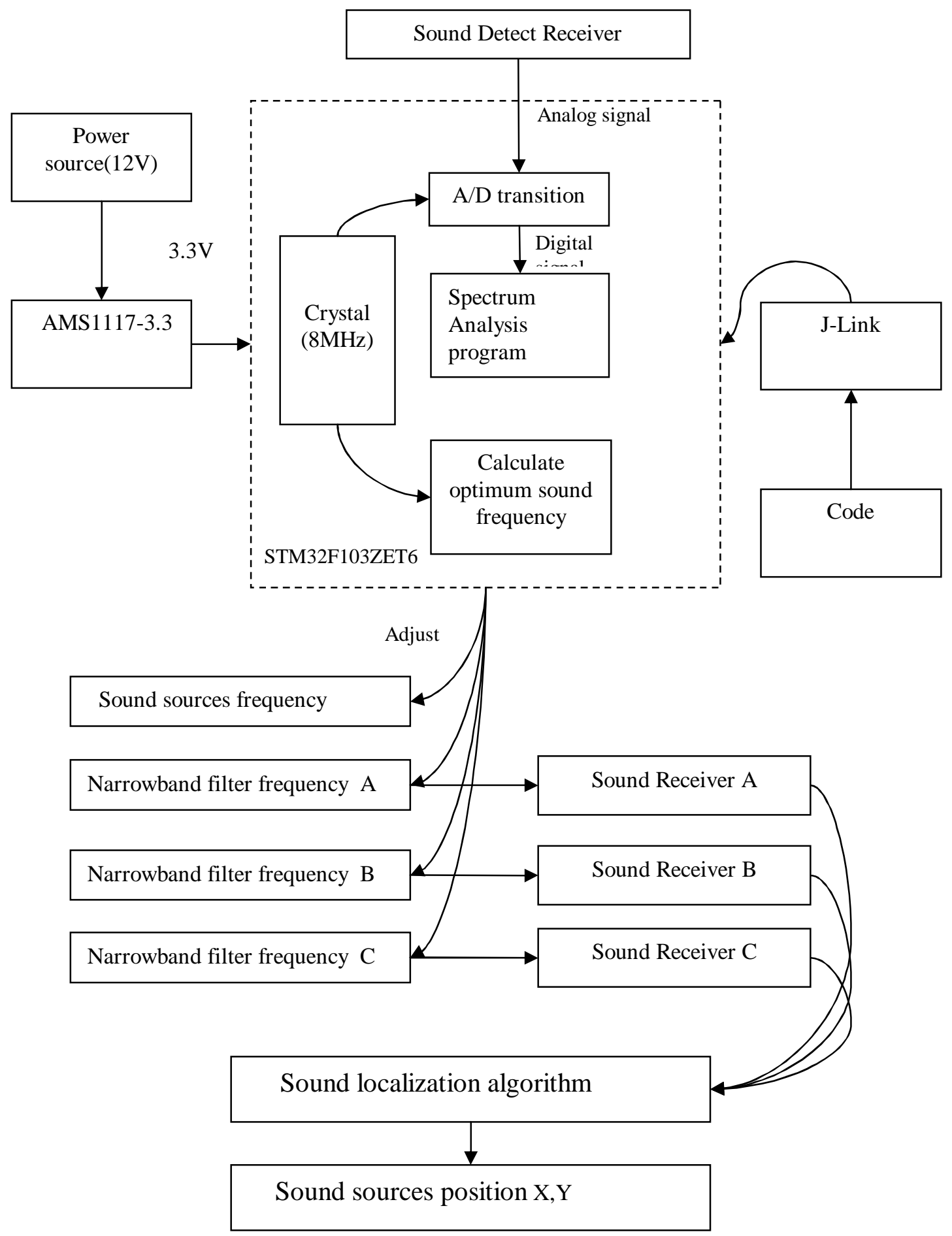

Fig. 3 The Sound positioning system diagram 
Fig. 3 shows the architecture of Sound positioning system, here are some details:

a. The ADCs of STM32F103ZET6 is a successive approximation A/D converter. Their maximum sampling frequency is $36 \mathrm{MHz}$, and the sampling frequency can be set according to the actual situation.

b. The power circuit is mainly composed of voltage stability chip AMS1117-3.3, it converts external voltage from $12 \mathrm{~V}$ to $3.3 \mathrm{~V}$, ensuring the normal work of data acquisition device.

c. The J-Link is used to download the code to STM32.

\section{Experiments}

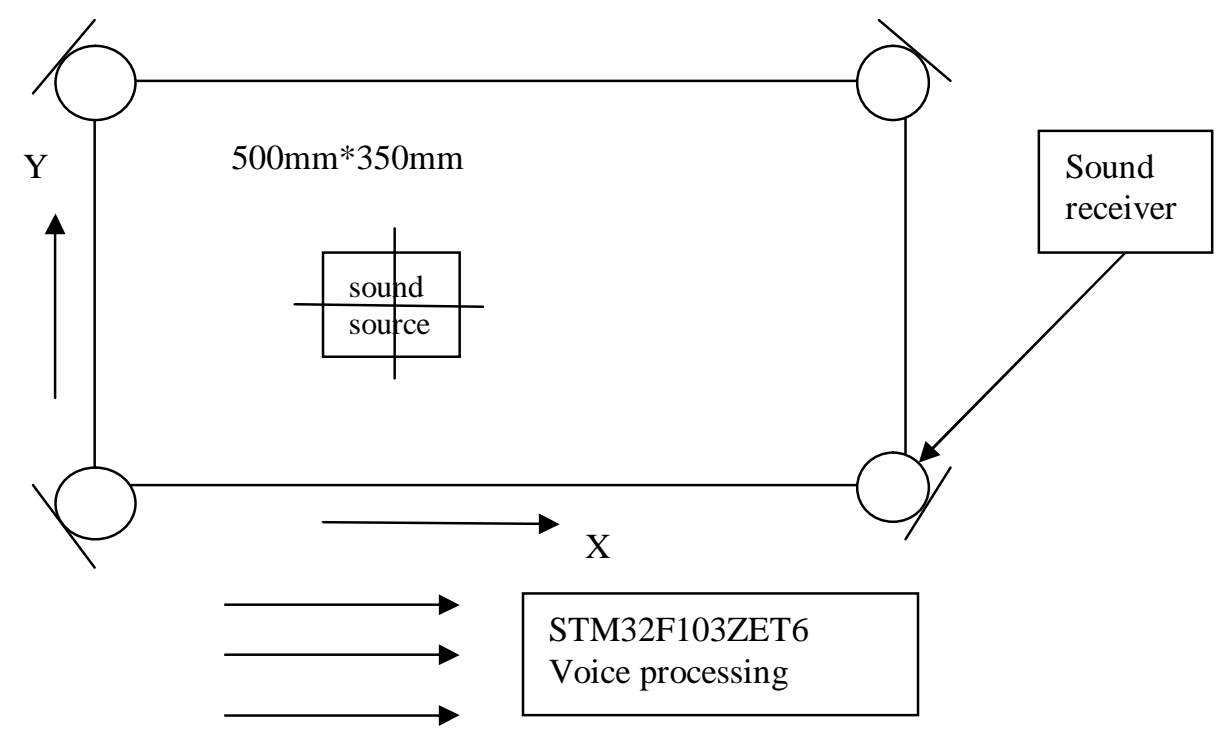

Fig.4 The experiment platform

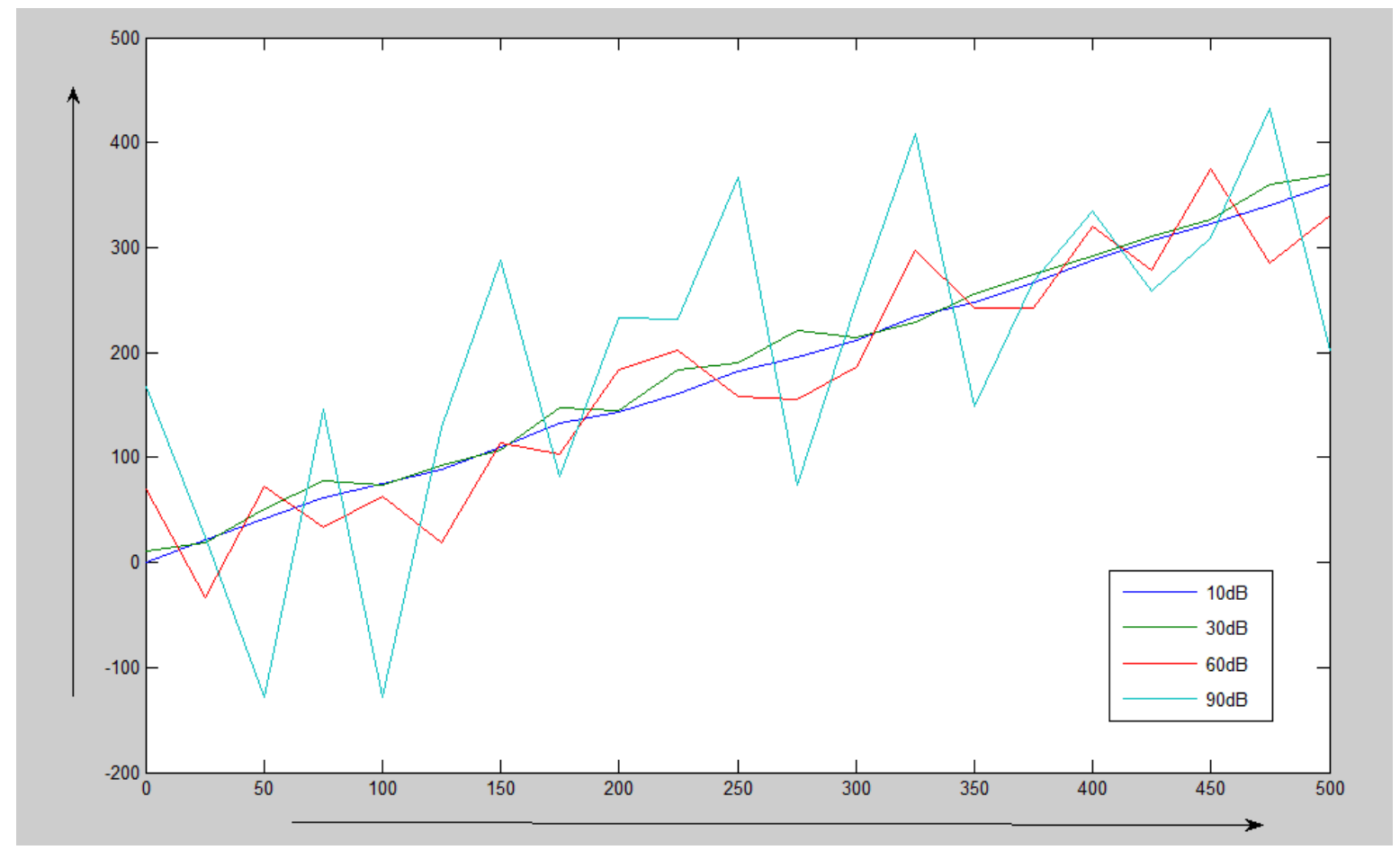

Fig.5 The experimental results under different noise environments 
To test the performance of the system, we established the experimental platform as shown in Fig.4: a piece of no more than $1 \mathrm{~m} 2$ tablet with a $500 \mathrm{~mm}$ x $350 \mathrm{~mm}$ paper, respectively fixed on the outside of the four corners to install a sound receiving module, receiving module through the wires to voice signal transmission to the information processing module, sound localization system based on sound module spreads through the air to the sound of voice signal receiving module, sound module location coordinates calculation.

Fig.5 shows the experimental results under different noise environments, here are some details:

a. Our test point positioning in $500 \mathrm{~mm} * 350 \mathrm{~mm}$ rectangular test area on the diagonal.

b. Under the background of $10 \mathrm{~dB}$ of environmental noise, the experiment test accuracy of anchor point is extremely high, less than one percent.

c. Under the background of $30 \mathrm{~dB}$ of environmental noise, the experiment testing anchor point with high accuracy, less than six percent.

d. Under the background of $60 \mathrm{~dB}$ environmental noise, the experiment test anchor point accurately, in an acceptable error range, less than ten percent.

e. Under the background of $90 \mathrm{~dB}$ of environmental noise, the experiment test anchor point accurately is poor, beyond the tolerance range, the system cannot work effectively.

f. Anyhow, sound positioning precision of the positioning system and decreased with the increase of environmental noise, the system by using frequency spectrum analysis, frequency selection techniques, which can withstand larger interference, thus under the $60 \mathrm{~dB}$ background of environmental noise, It work effectively

\section{Conclusions}

We have developed a sound localization system based on STM32, and introduces the optimal frequency spectrum detection and selection, as well as the localization algorithm. After that, we use experimental platform for sound localization experiment. Experimental tests show that the localization system can choose the optimal frequency and perform well in a complex noise environment, reflects the strong anti-interference ability.

\section{Acknowledgements}

Our works are supported by Natural Science Foundation of China (51407036) and Guangxi Science Foundation (2013GXNSFCA019019, 2014GXNSFBA118274)

\section{References}

[1] Luis Chaparro. Signals and Systems Using MATLAB (Second Edition) [M]. 2015, Pages 449-490.

[2] Glen Brisebois. Analog Circuit Design, Volume Three [M]. 2015, Pages 905-906.

[3] B. Apicellaa, A. Brunob, 1, X. Wangc, N. Spinellib. Fast Fourier Transform and autocorrelation function for the analysis of complex mass spectra, International Journal of Mass Spectrometry, [J], Volume 338, 15 March 2013, Pages 30-38.

[4] Douglas Self. Audio Engineering Explained- for professional audio recording [M]. 2015, 2010, Pages 267-294.

[5] Yilu Zhao, Xiong Chen, Bin Wang. Real-time sound source localization using hybrid framework. [J]. Applied Acoustics, Volume 74, Issue 12, December 2013, Pages 1367-1373. 